\title{
EVN \& MERLIN studies of a new sample of BL Lac objects
}

\author{
Hayley Bignall* \\ Joint Institute for VLBI in Europe \\ E-mail: bignallejive.nl
}

\section{Hermine Landt}

Harvard-Smithsonian Center for Astrophysics

E-mail: hlandt@head.cfa.harvard.edu

\section{Cormac Reynolds}

Joint Institute for VLBI in Europe

E-mail: reynolds@jive.nl

\section{Paolo Padovani}

European Southern Observatory

E-mail: Paolo.Padovani@eso.org

\section{Eric Perlman}

University of Maryland

E-mail: perlmanejca.umbc.edu

The recent Deep X-ray Radio Blazar Survey (DXRBS) has identified a sample of BL Lac objects spanning the intermediate range of spectral energy distributions between "classical" X-ray selected and radio-selected samples of BL Lacs. Detailed studies of such samples are needed to answer some of the currently open questions regarding the nature of BL Lacs and their place in a unified model of AGN. High-resolution radio imaging provides direct information on jet evolution and beaming parameters. We present some preliminary results from EVN \& MERLIN observations of sources in the DXRBS BL Lac sample for which little or no high-resolution radio data were previously available.

8th European VLBI Network Symposium

September 26-29, 2006

Toruń, Poland

\footnotetext{
* Speaker.
} 


\section{Introduction}

It is generally agreed that BL Lacertae objects (BL Lacs) are radio galaxies with their jets oriented close to our line of sight. Together, BL Lacs (which have weak or absent optical emission lines) and flat-spectrum quasars (with strong emission lines) are commonly called blazars. There are, however, a number of unresolved questions regarding the parent population of BL Lacs, the origin of their different spectral energy distributions (SEDs), and their relationship with flat-spectrum quasars. Radio observations at high angular resolution provide direct information on the structure and evolution of jets, which is important to help resolve some of the outstanding problems with unified models for AGN.

Our current knowledge of BL Lacs is based mainly on studies of two samples: the radioselected 1-Jy sample[1] and the X-ray selected Einstein Medium Sensitivity Survey (EMSS) sample[2]. These two surveys, with their widely different selection bands and relatively high flux limits, introduced a dichotomy in the class of BL Lacs, with the sources found by each survey having distinctly different properties. The EMSS had no radio selection criteria, and its BL Lacs were found to have low extended radio powers and morphologies typical of Fanaroff-Riley type I (FR I) radio galaxies [3], 四]. For some 1-Jy BL Lacs, however, FR II-like properties have been reported, suggesting that these sources may be the beamed counterparts of more powerful radio galaxies, and hence more closely related to flat-spectrum quasars than the X-ray selected BL Lacs [5]-[7]. The EMSS found only BL Lacs with broad-band emission properties indicative of high-energy (UV/soft Xray) synchrotron emission peaks, whereas the majority of sources selected by the 1-Jy survey were low-energy (IR/optical) peaked BL Lacs.

It is not yet clear if the observed differences are predominantly due to different viewing angles and a frequency dependence of relativistic beaming ([8], [9]), or if the jets have a large range of intrinsic synchrotron emission peak frequencies, $v_{\text {peak }}[10]$. Finding an answer is relevant for the physics of jet formation. If orientation plays a role, it would imply that low- and high-energy synchrotron emission are produced at different locations in the jet. On the other hand, if BL Lacs have jets with a large range of $v_{\text {peak }}$, it would follow that physical properties around black holes in AGN, e.g. maximum electron energy and magnetic field strength, can have a very large spread.

\section{The classification of BL Lacs and the DXRBS BL Lac sample}

Recently, the criteria used to classify radio sources as BL Lacs have been questioned. Marchã et al. [11] proposed revised criteria for separating blazars (beamed objects) from radio galaxies, based on the Ca H\&K break (a stellar absorption feature) in the optical spectrum, as well as separating BL Lacs from flat-spectrum quasars based on the equivalent width of emission lines. The problem of blazar classification was re-addressed by Landt et al. [12], who argued that all radioloud AGN can be effectively separated into intrinsically weak- and strong-lined sources based on the narrow $\left[\mathrm{O}_{\mathrm{III}}\right]$ emission line.

In order to disentangle the effects of different viewing angles, intrinsic jet power and environment of the AGN host galaxies, all of which influence the observed properties of radio sources, it is important to compare sources which have been selected in a uniform way. Recently, new samples of BL Lacs have been found by cross-correlating existing radio and X-ray source catalogues, 
searching for compact sources which are then classified based on optical identification. These searches tend to find BL Lacs with properties intermediate between those of the EMSS and 1-Jy samples, providing strong evidence that there is in fact a continuum of BL Lac SEDs, and that the observed dichotomy is simply a product of selection effects of the earlier surveys. Detailed studies of intermediate samples are expected to provide a more complete picture of the BL Lac class of objects and their relation to radio galaxies. One such sample was drawn from the Deep X-ray Radio Blazar Survey (DXRBS).

The details of the DXRBS sample selection are described in [13] and [14]. Briefly, the sample was selected by cross-correlating the WGACAT [15] of "serendipitous" X-ray sources detected by the ROSAT Position Sensitive Proportional Counter (PSPC) with several publicly available radio catalogues. A radio spectral index cut was applied to reject most steep-spectrum sources, since the aim of the DXRBS was to find beamed objects, so-called blazars, that are dominated by a flat-spectrum radio core.

The majority of DXRBS sources are strong emission-line quasars, but a sizeable number - 45 sources - are classified as BL Lacs according to the criteria of Marchã et al. [11]. Of the DXRBS BL Lacs, 11 had already been identified in earlier surveys and the rest were newly discovered. The DXRBS BL Lac sample is comparable in size to the 34 sources of the 1-Jy and 44 sources of the EMSS BL Lac samples. The DXRBS sample contains roughly equal numbers of low- $\left(\alpha_{\mathrm{rx}}>0.78\right.$; 26 sources) and high-energy peaked BL Lacs ( $\alpha_{\mathrm{rx}} \leq 0.78 ; 19$ sources).

All DXRBS sources classified as BL Lacs have been subsequently observed with the VLA at $20 \mathrm{~cm}$ in A and C configurations, and with the ATCA for the sources with $\delta<-40^{\circ}$ (Landt et al., 2007, in preparation). More sensitive optical spectroscopy is also being undertaken for the sources for which we lack firm redshift measurements.

Of the northern DXRBS BL Lacs, 16 sources had little or no VLBI data available in the literature or archives as of early 2005, so EVN and MERLIN observations were proposed. VLBI observations are important to investigate relativistic beaming and account for this in comparing BL Lacs with radio galaxies.

\section{EVN and MERLIN observations}

Combined EVN and MERLIN observations were undertaken during 3-5 June 2005. The 16 target sources were observed in a 48-hr period (one additional source was in the target list, but was not scheduled due to its position being close to the Sun in early June). The observations were carried out at $1.6 \mathrm{GHz}$, with the VLBI data recorded at $128 \mathrm{Mbps}$ in two 8-MHz subbands. Full polarization information was obtained, but only total intensity data are presented here. Phase-referencing was used for 11 of the target sources, which either had arcsecond positional uncertainties or the peak flux density in VLA A-conf. data was substantially lower than $100 \mathrm{mJy}$. Initial processing of MERLIN data was done with local software at Jodrell Bank Observatory, and standard procedures in AIPS were used for subsequent processing of both MERLIN and EVN data.

The flux density scale of the MERLIN data is tied to 3C286, which is also used to calibrate the linear polarization position angle. The flux density of OQ208 in our MERLIN data was found to be $1.14 \mathrm{Jy}$, and MERLIN data for all other sources were calibrated based on this value. The EVN data were calibrated independently based on measured gain curves and system temperatures. 


\begin{tabular}{lcrrrrr}
\hline \hline X-ray catalogue name & Redshift & $\begin{array}{r}S_{\mathrm{VLA}}^{\text {peak }} \\
{[\mathrm{mJy} / \mathrm{beam}]}\end{array}$ & $\begin{array}{r}S_{\mathrm{MER}}^{\text {tot }} \\
{[\mathrm{mJy}]}\end{array}$ & $\begin{array}{r}S_{\mathrm{MER}}^{\text {peak }} \\
{[\mathrm{mJy} / \mathrm{beam}]}\end{array}$ & $\begin{array}{r}S_{\mathrm{EVN}}^{\text {tot }} \\
{[\mathrm{mJy}]}\end{array}$ & $\begin{array}{r}S_{\mathrm{EVN}}^{\text {peak }} \\
{[\mathrm{mJy} / \mathrm{beam}]}\end{array}$ \\
\hline WGAJ0245+1047 & 0.070 & 29 & 36 & 35 & 33 & 24 \\
WGAJ0313+4115 & 0.029 & 55 & 97 & 44 & 41 & 19 \\
WGAJ0558+5328 & 0.036 & 297 & 296 & 95 & 39 & 22 \\
WGAJ0847+1133 & 0.199 & & 34 & 21 & 11 & 11 \\
RXJ09168+523 & 0.190 & 58 & 60 & 52 & 49 & 29 \\
WGAJ0940+2603 & $\ldots$ & 372 & & & 427 & 355 \\
WGA1012+06 & 0.727 & & 300 & 115 & 97 & 69 \\
WGA1202+44 & $\ldots$ & & 61 & 50 & 34 & 25 \\
WGAJ1204-0710 & 0.185 & & 140 & 99 & 67 & 48 \\
WGAJ1231+2848 & 1.000 & 86 & 94 & 78 & 65 & 32 \\
WGAJ1311-0521 & 0.160 & 28 & & & 18 & 18 \\
WGAJ1320+0140 & 1.235 & 497 & & & 471 & 138 \\
WGAJ1539-0658 & $\ldots$ & 44 & 63 & 62 & 55 & 48 \\
WGAJ1744-0517 & 0.310 & & & 14 & 11 & 11 \\
EXO1811+3143 & 0.117 & 103 & 117 & 105 & 91 & 80 \\
WGAJ1840+5452 & 0.646 & 102 & 114 & 88 & 95 & 82 \\
\hline
\end{tabular}

Table 1: The DXRBS BL Lacs observed with EVN and MERLIN at 1.6 GHz. Peak and total flux densities from preliminary analysis of EVN and MERLIN data are shown, along with source redshifts and peak flux densities from VLA A configuration data at $1.4 \mathrm{GHz}$ (where available).

No combined calibration or analysis of EVN and MERLIN data has yet been done for the data presented here.

The observed sources (listed here by their associated X-ray catalogue counterparts), redshifts, and peak flux densities (where available) from 1.4-GHz VLA data in A configuration are listed in Table 1, along with peak and total flux densities from MERLIN and EVN images at 1.6 GHz. The EVN and MERLIN results presented are based on preliminary images made in Difmap, using uniform weighting, and iterative CLEAN and self-calibration. All sources are clearly detected. Note that the VLA data, as well as being at a slightly lower frequency, are not simultaneous with the EVN+MERLIN data and the sources may be variable. Although the EVN and MERLIN results are very preliminary, the values in Table 1 give an indication of the distribution of emission on different angular scales. There is clearly a wide variation in radio properties of the different sources within this sample. The typical angular resolution for the data from each array is 1-2 arcseconds for the VLA in A configuration, 0.1-0.2 arcseconds for MERLIN, and $\sim 3$ mas for the EVN. All of the data now available will allow us to investigate source structure from milliarcsecond to arcminute angular scales, corresponding to a range from parsec scales out to hundreds of kpc (depending on source redshift), where often large changes in jet orientation and collimation are observed as the jet evolves and interacts with the surrounding medium.

VLA images for one source in the sample, RX J0916.8+5238, are displayed in Figure 1 and preliminary EVN and MERLIN images are displayed in Figure \&. This object had already been 

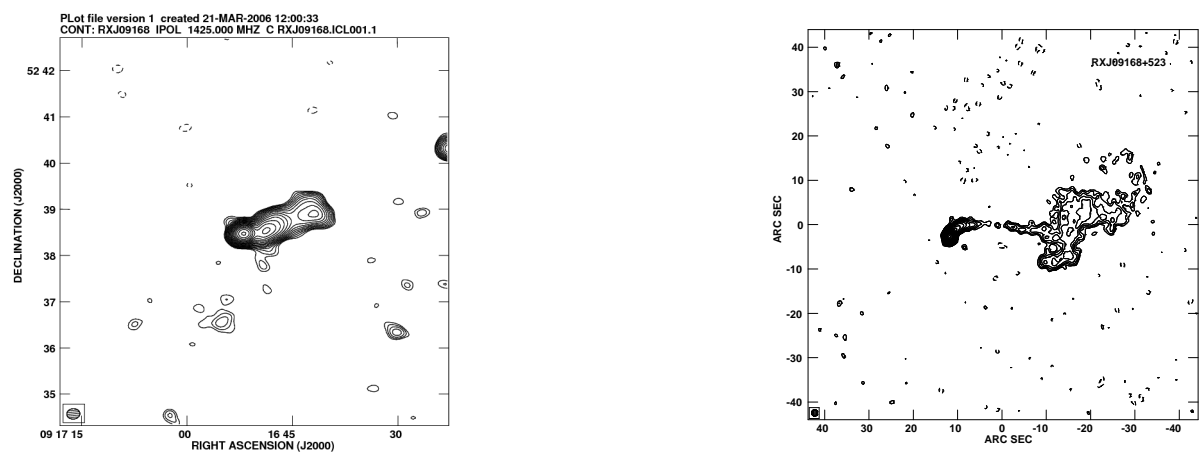

Figure 1: RX J0916.8+5238 as observed with the VLA in C configuration (left) and A configuration (right) at $1.4 \mathrm{GHz}$. The residual $\mathrm{rms}$ for each image is $\sigma=0.06 \mathrm{mJy} / \mathrm{beam}$ and $\sigma=0.02 \mathrm{mJy} / \mathrm{beam}$, respectively, and contours are shown at $\left(-1,1, \sqrt{2}^{n}\right) \times 3 \sigma$, where $n=1,2,3 \ldots$ up to the maximum in the image. The peak values are $69 \mathrm{mJy} /$ beam for the left-hand image and $58 \mathrm{mJy} / \mathrm{beam}$ for the right-hand image.
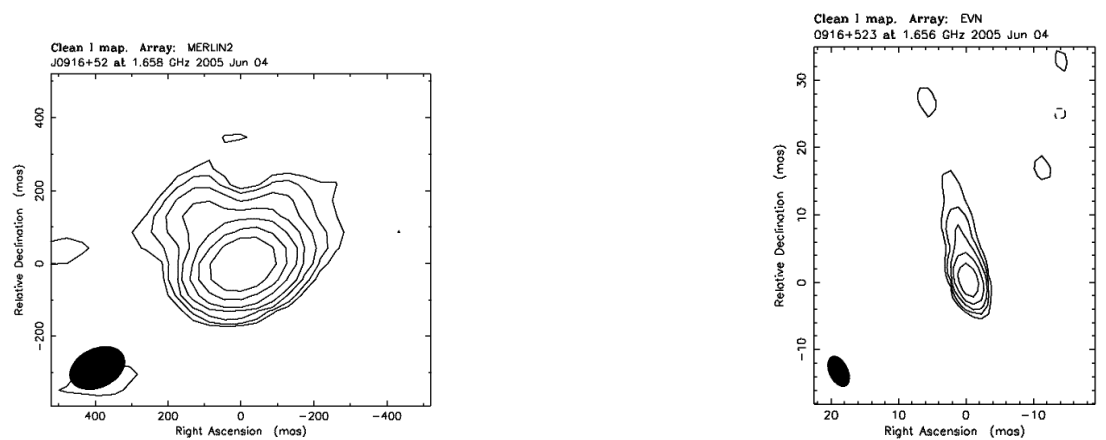

Figure 2: RX J0916.8+5238 as observed with MERLIN (left) and the EVN (right) at 1.6 GHz. The MERLIN image peak is $52 \mathrm{mJy} / \mathrm{beam}$, and contours are shown at $(1,2,4,8,16,32,64) \times 0.3 \mathrm{mJy} / \mathrm{beam}$. The EVN image peak is $29 \mathrm{mJy} / \mathrm{beam}$, and contours are shown at $(-1,1,2,4,18,16) \times 1 \mathrm{mJy} / \mathrm{beam}$. The residual rms for these preliminary images is $\sim 0.2 \mathrm{mJy} / \mathrm{beam}$.

identified as a BL Lac at redshift $z=0.19$ from the ROSAT All-Sky Survey by Nass et al. [16] prior to the DXRBS. Assuming a WMAP-based cosmology, an angular scale of 1 arcsecond translates to $3 \mathrm{kpc}$ at the source. The images show the complex extended structure and large misalignment of the jet in this case between pc and kpc scales.

\section{Summary}

We have observed 16 BL Lac objects identified in the Deep X-ray Radio Blazar Survey (DXRBS) with the EVN and MERLIN at $18 \mathrm{~cm}$. Combined with 20-cm VLA data and other available VLBI data for the entire sample, these data will be useful for investigating the radio properties of different types of BL Lacs selected in a uniform way and spanning the intermediate range between high- and low-energy peaked BL Lacs. 


\section{Acknowledgments}

The European VLBI Network is a joint facility of European, Chinese, South African and other radio astronomy institutes funded by their national research councils. MERLIN is a National Facility operated by the University of Manchester at Jodrell Bank Observatory on behalf of PPARC. The VLA is operated by the National Radio Astronomy Observatory, a facility of the National Science Foundation operated under cooperative agreement by Associated Universities, Inc.

\section{References}

[1] M. Stickel, J.W. Fried, H. Kuehr, P. Padovani and C.M. Urry, The optical identification status of the 1 Jy radio source catalogue, A\&AS 374 (1991) 211.

[2] S.L. Morris et al., The luminosity function and cosmological evolution of X-ray-selected BL Lacertae objects, ApJ 380 (1991) 49.

[3] E.S. Perlman and J.T. Stocke, The radio structure source of X-ray-selected BL Lacertae objects, ApJ 406 (1993) 430.

[4] T.A. Rector, J.T. Stocke, E.S. Perlman, S.L. Morris and I.M. Gioia, The properties of the $X$-ray-selected EMSS sample of BL Lacertae objects, AJ 120 (2000) 1626.

[5] R.T. Kollgaard, J.F.C. Wardle, D.H. Roberts and D.C. Gabuzda, Radio constraints on the nature of BL Lacertae objects and their parent population, AJ 104 (1992) 1687.

[6] P. Cassaro, C. Stanghellini, M. Bondi, D. Dallacasa, R. della Ceca, and R.A. Zappalà, Extended radio emission in BL Lac objects. I. The images, A\&AS 139 (1999) 601.

[7] T.A. Rector and J.T. Stocke, The properties of the radio-selected 1 Jy sample of BL Lacertae objects, AJ 122 (2001) 565.

[8] L. Maraschi, L. G. Ghisellini, E.G. Tanzi and A. Treves, Spectral properties of blazars. II. an X-Ray observed sample, ApJ 310 (1986) 325.

[9] M. Georganopoulos and A.P. Marscher, A viewing angle-kinetic luminosity unification scheme for BL Lacertae objects, ApJ 506 (1998) 621.

[10] P. Padovani and P. Giomi, The connection between X-ray-and radio-selected BL Lacertae objects, ApJ 444 (1995) 567.

[11] M.J.M. Marcha, I.W.A. Browne, C.D. Impey and P.S. Smith, Optical spectroscopy and polarization of a new sample of optically bright flat radio spectrum sources, MNRAS 281 (1996) 425.

[12] H. Landt, P. Padovani, E.S. Perlman and P. Giommi, A physical classification scheme for blazars, MNRAS 351 (2004) 83.

[13] E.S. Perlman et al., The Deep X-ray Radio Blazar Survey. I. Methods and first results, AJ 115 (1998) 1253.

[14] H. Landt, P. Padovani, E.S. Perlman, P. Giommi, H. Bignall and A. Tzioumis, The Deep X-ray Radio Blazar Survey (DXRBS) - II. New identifications, MNRAS 323 (2001) 757.

[15] N.E. White, P. Giommi and L. Angelini, The WGA catalog of ROSAT point sources, BAAS (1994) 1372 [http://wgacat.gsfc.nasa.gov/wgacat/wgacat.html]

[16] P. Nass, N. Bade, R.I. Kollgaard, S.A. Laurent-Muehleisen, D. Reimers and W. Voges, BL Lacertae objects in the ROSAT All-Sky Survey: new objects and comparison of different search techniques., A\&A 309 (1996) 419. 